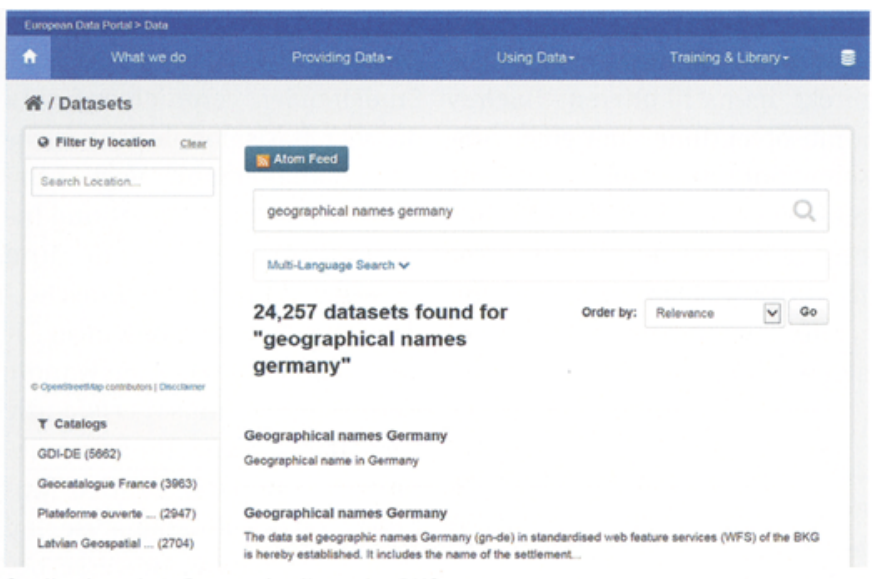

Quelle: Aus einer Pressemitteilung des BKG

zen und -diensten “ (bzw. zur Datenspezifikation geographischer Namen, Annex I) der europäischen Geodateninfrastruktur hergestellt. Damit ist das BKG eine der ersten Institutionen in Europa, die einen nationalen Namendatendienst INSPIRE-konform zur Verfügung stellt. Gemäß INSPIRE-Zeitplan ist diese Verpflichtung erst im Jahr 2017 notwendig.

Die Vermessungs- und Katasterverwaltungen der Länder, die statistischen Landesämter und das Statistische Bundesamt, private und universitäre Institutionen für Sprache und Kultur etc. sind für INSPIRE ebenfalls potenziell „datenhaltende Stellen“ (i. S. d. Geodatenzugangsgesetzes - GeoZG) für geographische $\mathrm{Na}$ mendaten, allerdings mit bisher sehr unterschiedlicher Namensdichte oder-vielfalt.

Beachtet man die Mehrsprachigkeit und den kulturellen Reichtum in Europa, so verwenden die Menschen oftmals unterschiedliche Schreibweisen und Sprachen, wenn sie über denselben Ort sprechen. Selbst innerhalb eines Landes wird teilweise mehr als eine (offizielle) Schreibweise benutzt. Diese von INSPIRE unterstützten Prinzipien unterstreichen auch die Resolutionen der Vereinten Nationen zur Standardisierung geographischer Namen, welche sich in Deutschland in den Arbeiten des Ständigen Ausschusses für geographische $\mathrm{Na}-$ men (StAGN) widerspiegeln. schaft (DFG) hat dem Antrag der Staatsbibliothek zu Berlin (SBB) zur Einrichtung eines Fachinformationsdienstes Kartographie und Geobasisdaten zugestimmt. In den nächsten drei Jahren wird die Kartenabteilung der SBB neue Serviceangebote aufsetzen, die insbesondere elektronische Angebote beinhalten. Im nächsten Heft der $\mathrm{KN}$ wird ausführlicher berichtet.

Wolfgang Crom,

Staatsbibliothek zu Berlin

\section{Eine neue Sektion „Öko- und Kartosemiotik"}

Das IT-Zentrum der Universität Passau war am 28. November 2015 Gastgeber für die Deutsche
Gesellschaft für Semiotik (DGS), wo die Beiratssitzung und danach die Mitgliederversammlung der DGS stattfanden. Die DGS befindet sich zurzeit auf Kurs einer Konsolidierung und Erneuerung.

Für die Zeitperiode 2015-2017 wurden 18 Sektionen der DGS vorgeschlagen und gewählt. Eine der neuen Sektionen ist die Sektion "Öko- und Kartosemiotik“. Sie verbindet zwei angewandte Semiotiken - Ökosemiotik und Kartosemiotik - und strebt somit eine anwendungsorientiertere und multidisziplinäre Ausrichtung an. Die Sektion wird von Monika Huch und Alexander Wolodtschenko geleitet (http:// www.semiotik.eu/Oeko-undKartosemiotik).

Die neuen öko-/kartosemiotischen Aufgaben der Sektion orientieren sich an theoretischmethodischen und angewandtpraktischen Richtungen mit der Anwendung von öko-/kartosemiotischen Produkten auf mobilen Geräten und die Einbeziehung der elektronischen Medien, z. B. in Form von e-Journals, multidisziplinären und multimedialen (Mini-)Atlanten u. ä. Visualisierungen.

A. Wolodtschenko, Dresden

\section{Symposium der „International Society for the History of the Map" (ISHMap)}

Lissabon am 3./4. Juni 2016

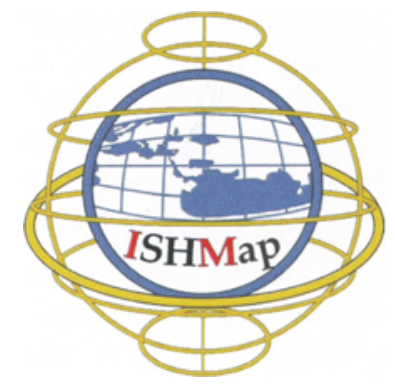

Die „International Society for the History of the Map“ (ISHMap, vgl. http://ishm.elte.hu/) wurde

2011 in London mit dem Ziel gegründet, eine Plattform für die Geschichte der Kartographie zu schaffen und die Gemeinschaft aller Forschenden international zu vertreten. ISHMap ist die erste und bislang einzige internationale akademische Gesellschaft, die das Studium der Kartographiegeschichte in allen Gesellschaften und zu allen historischen Zeiten fördert, indem sie die Ausbildung des Faches, die Kommunikation zwischen den Mitgliedern und allen anderen Interessierten sowie die Forschung, die Lehre und die Finanzierung des Faches „Geschichte der Kartographie“ unterstützt.

Die Gesellschaft wird derzeit von der auf der jährlichen Generalversammlung am 11. Juli 2015 in Antwerpen gewählten britischen Historikerin Sarah Tyacke (London/UK, frühere Geschäftsführerin der Nationalarchive von England und Wales) als Präsidentin geleitet. Zur Generalsekretärin wurde Frau Merve Senem Arkam (Doktoratsstudentin, Budapest/ Ungarn) bestellt.

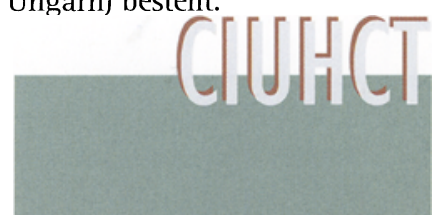

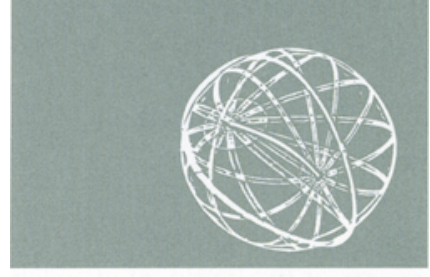

Centro Interuniversitánio de Histónia das Ciências e da Tecnologia F C UL I F C . UN L

Als Trustees der Gesellschaft fungieren Dr. Catherine DelanoSmith (UK, Herausgeberin der internationalen Fachzeitschrift „Imago Mundi“, London/UK), Prof. Dr. Gilles Palsky (Institut für Geographie, Paris/Frankreich), Prof. Dr. Junia Furtado (Universidade Federal de Minas Gerais/ Brasilien), Prof. Dr. Matthew Edney (University of Southern Maine und Direktor des internationalen Projektes „The History 